\title{
Monte Carlo Calculation of High Frequency Mobility and Diffusion Noise in Nitride-Based Semiconductors
}

\author{
E. Starikov ${ }^{a, b}$, P. Shiktorov ${ }^{a}$, V. Gružinskis ${ }^{a}$, L. VArAni $^{b}$, \\ J.C. VAissière ${ }^{b}$, C. PAlermo ${ }^{b}$ AND L. RegGiani ${ }^{c}$ \\ ${ }^{a}$ Semiconductor Physics Institute, A. Goštauto 11, 2600 Vilnius, Lithuania \\ ${ }^{b}$ CEM2 - Centre d'Electronique et de Micro-optoelectronique de Montpellier \\ (CNRS UMR 5507) Université Montpellier II, 34095 Montpellier Cedex 5, France \\ ${ }^{c}$ INFM - National Nanotechnology Laboratory, Dipartimento di Ingegneria \\ dell'Innovazione, Università di Lecce, via Arnesano s/n, 73100 Lecce, Italy
}

Monte Carlo simulations of high-field transport in semiconductor nitrides, GaN and $\mathrm{InN}$, is used to calculate the velocity-field characteristics and the high-frequency behavior of the differential mobility, spectral density of velocity fluctuations, and noise temperature. It is found that due to very short relaxation time scales of nitrides, the characteristic frequencies associated with extrema and cutoff decay of the negative differential mobility, etc. are shifted to higher frequency range with respect to the case of standard $A_{3} B_{5}$ compounds. This property is favorable for applications of nitrides in the $\mathrm{THz}$ frequency range.

PACS numbers: $72.20 . \mathrm{Ht}, 72.30 .+\mathrm{q}, 72.70 .+\mathrm{m}$

\section{Introduction}

Recently an increasing interest has been paid to wide bandgap nitride-based materials, namely GaN, InN, AlN, as well as to various nitrides-based structures and devices. Such an interest is motivated by the promising properties that nitrides offer for micro- and opto-electronic applications. As a consequence, the need of realistic models able to predict and analyze the electrical and microwave properties of bulk nitrides and their structures is a mandatory issue. Accordingly, main attention was addressed to the static low-field electron mobility and velocity-field relation (see, e.g. [1-3] and references therein), while theoretical considerations of 
the high-frequency behavior of the differential mobility and electronic noise of hot carriers remained practically absent.

The aim of this paper is to fill this lack of knowledge by performing Monte Carlo (MC) simulations to obtain the differential mobility and velocity noise characteristics in wurtzite GaN and InN. For this sake, linear response functions of the longitudinal velocity and differential mobility spectra $\mu(\omega)$ are calculated by using the technique based on the velocity average over before- and after-scattering ensembles [4] when a constant electric field $E$ is applied to the bulk material. For the noise analysis, the correlation function of longitudinal velocity fluctuations, $C_{v v}(s)$, is calculated and the spectral density of velocity fluctuations, $S_{v v}(\omega)$ is then obtained by Fourier transform of $C_{v v}(s)$. Then, the noise temperature and its spectrum is obtained as $T_{n}(\omega)=e S_{v v}(\omega) /\left(4 k_{\mathrm{B}} \operatorname{Re}[\mu(\omega)]\right)$.

\section{Numerical results}

Figure 1a compares the velocity-field characteristics calculated for $\mathrm{InN}$ and GaN at lattice temperature $T=300 \mathrm{~K}$ and doping level $N_{\mathrm{D}}=10^{17} \mathrm{~cm}^{-3}$. Here models labelled as "1" correspond to Ref. [2], while the model 2 assumes the smaller values of the energy separation and efective mass for the second valley $\left(\Delta_{12}=400 \mathrm{meV}\right.$ and $\left.m_{2}^{*}=0.4\right)$. For comparison, Fig. 1a also reports the exper-
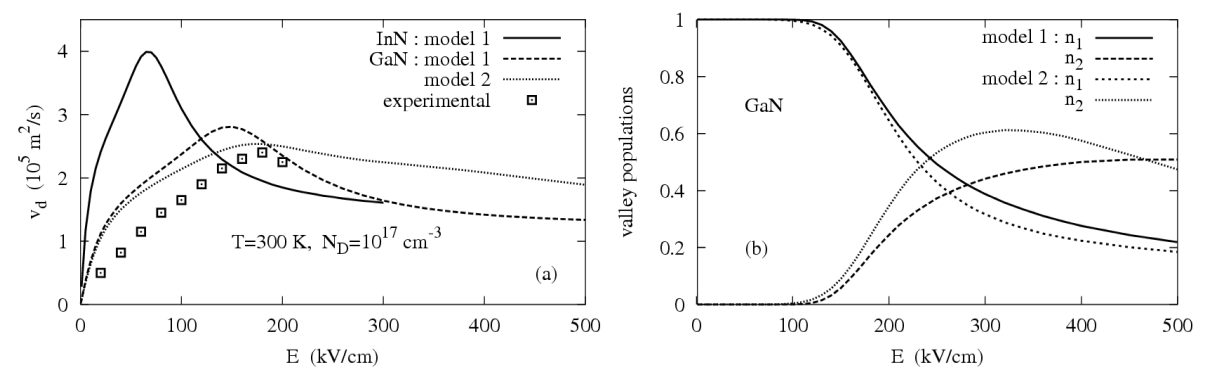

Fig. 1. Field dependence of: (a) the drift velocity of $\mathrm{InN}$ and GaN and (b) relative populations of the lowest and second valleys.

imental data of Ref. [3]. As follows from Fig. 1a, by using the reduced values of $\Delta_{12}$ and $m_{2}^{*}$ and keeping other parameters similar to those of model 1 , the velocity peak is smoothed out, and the agreement with the experiment is significantly improved. As follows from Fig. 1b, which compares field dependences of the relative populations of the lowest (1) and second valleys calculated for two models of GaN, in all cases the appearance of the negative differential mobility (NDM) is directly related with electron transitions to the upper valleys.

Similarly to the standard $A_{3} B_{5}$ compounds, we have found that under hot-carrier conditions the linear response and correlation functions exhibit pronounced negative tail caused mainly by electron transfer between nonequivalent valleys. As 

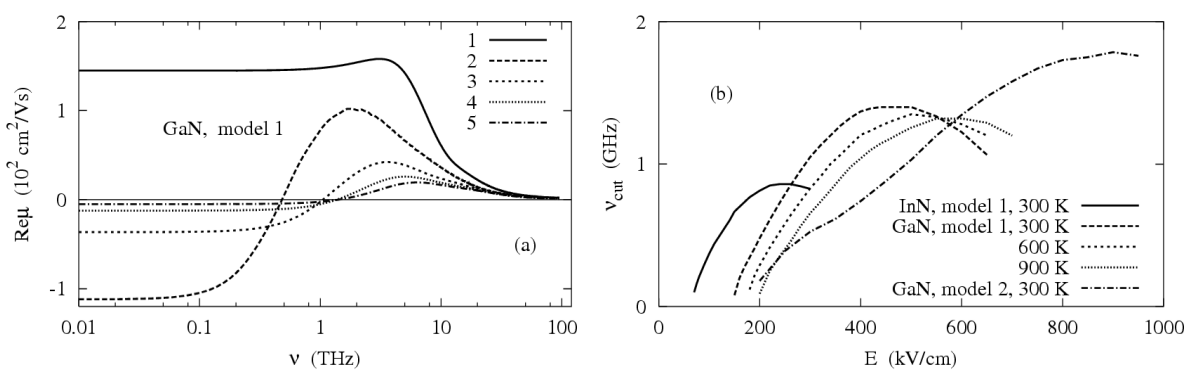

Fig. 2. (a) Frequency dependence of the real part of the differential mobility and (b) field dependence of the NDM cutoff frequency.

a result, in all cases considered here, the hot-carrier differential mobility $\operatorname{Re}[\mu(\nu)]$ exhibits a plateau in the low-frequency region (up to about $0.1 \sim 1 \mathrm{THz}$ depending on the value of $E$ ), a peak at intermediate frequencies $(\nu=1 \sim 10 \mathrm{THz})$, and a $1 / \nu^{2}$ decay at the highest frequencies $(\nu \geq 10 \mathrm{THz})$. As an example, Fig. 2a shows $\operatorname{Re}[\mu(\nu)]$ spectra calculated by the MC approach for the model 1 of GaN.

For the use of nitrides as active elements in electronic devices, the maximum value of the NDM cutoff frequency, $\nu_{\text {cut }}$, is of special interest. Accordingly, the field dependence of the NDM cutoff frequency calculated for InN and GaN (models 1 and 2) are summarized in Fig. 2b. To illustrate the possible modifications of $\nu_{\text {cut }}-E$ relation with the lattice temperature, the results for GaN (model 1) are presented at three different temperatures. As follows from Fig. 2b, the general behavior of the $\nu_{\text {cut }}-E$ relation is quite similar in all the cases considered. The NDM cutoff frequency starts from zero at the threshold field $E_{\mathrm{th}}$, then increases with $E$ and reaches a maximum value at sufficiently high electric fields in the range of $E=(3 \sim 5) E_{\mathrm{th}}$. The high value of the NDM cutoff frequency even at sufficiently high temperatures suggests that $\mathrm{InN}$ and GaN are promising materials for high-frequency and high-power applications in a wide range of temperatures.

The spectral behavior of velocity noise is illustrated by Fig. 3. Figure 3a presents the spectral density of velocity fluctuations, $S_{v v}(\nu)$, calculated by MC
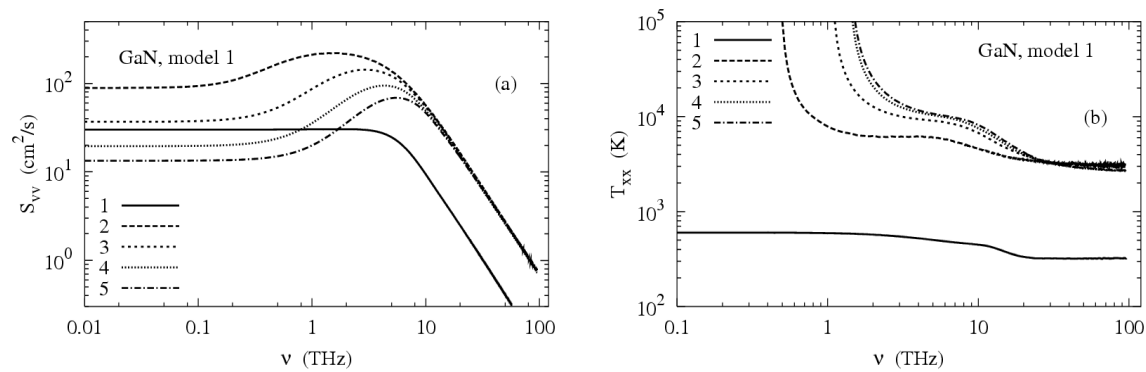

Fig. 3. Frequency dependence of: (a) the spectral density of velocity fluctuations and (b) the noise temperature. 
method for GaN (model 1) at $300 \mathrm{~K}$. By comparing Figs. 2a and 3a, we conclude that the spectral behavior of $\operatorname{Re}[\mu(\nu)]$ and $S_{v v}(\nu)$ is rather similar. They both start with a low-frequency plateau, exhibit a maximum and vanish with a $1 / \nu^{2}$ slope in more or less the same frequency regions related with the same dynamics and relaxation processes (heating and transfer to upper valleys, momentum and energy relaxation, etc.). By comparing with the case of standard III-V compounds, we concludes that due to sufficiently short characteristic times in nitrides, main features of $\operatorname{Re}[\mu(\nu)]$ and $S_{v v}(\nu)$ spectra (positions of extrema, cutoff decay of the NDM, etc.) are shifted into the higher frequency range what is favorable for highfrequency applications of nitrides.

Another relevant quantity to characterize the high-frequency behavior of hot-carriers is the noise temperature. The frequency and field dependences of this quantity can be obtained by using the differential mobility and noise spectra calculated above. Accordingly, Fig. 3b shows the noise temperature spectra calculated by the MC method for the model 1 of GaN at different values of the electric field. Below the threshold field for the NDM onset, both $\operatorname{Re}[\mu(\nu)]$ and $S_{v v}(\nu)$ are positive, so that $T_{n}(\nu)$ is a positively defined function, and, hence, it can be experimentally measured through the output noise power (usually as a function of $E$ at some fixed frequency). As follows from Fig. 3b, in this case (see curve 1) the noise temperature is practically independent of frequency in the sub- and near- $\mathrm{THz}$ region where the frequency dependences of $\operatorname{Re}[\mu(\nu)]$ and $S_{v v}(\nu)$, being governed by the same relaxation rates, compensate in part each other. With the increase in $E$, an extra increase in $T_{n}(\nu)$ appears in the low-frequency region (mostly due to the decrease of the differential mobility). Finally, for fields above the NDM threshold value the low-frequency $T_{n}(\nu)$ becomes undefined and takes positive values above the NDM cutoff frequency only.

\section{Acknowledgments}

Authors acknowledge the financial support of NATO Collaborative Linkage Grant PST.EAP.CLG 980629, the French CNRS position of associated researcher and of the Italian Ministry of Education, University and Research (MIUR) under the project "Noise Models and Measurements in Nanostructures".

\section{References}

[1] S. Dhar, S. Ghosh, J. Appl. Phys 86, 2668 (1999).

[2] B. Foutz, S. O'Leary, M. Shur, L. Eastman, J. Appl. Phys 85, 7727 (1999).

[3] J.M. Barker, R. Akis, T.J. Thornton, D.K. Ferry, S.M. Goodnick, Phys. Status Solidi A 190, 263 (2002).

[4] E. Starikov, P. Shiktorov, V. Gružinskis, L. Reggiani, L. Varani, J.C. Vaissière, Jian H. Zhao, J. Appl. Phys 89, 1161 (2001). 